\title{
Teaching thermodynamic principles from industry ecology bionics knowledge aspects
}

\author{
Hongxu LI \\ School of metallurgical and ecological engineering , University of science and technology, Beijing \\ 100083, China \\ email: lihongxu@ustb.edu.cn
}

Keywords: Thermodynamics; teaching; professionals; industrial process;ecosystem

\begin{abstract}
Thermodynamics is a branch of natural science concerned with heat and its relation to energy and work. It applies to a wide variety of topics in science and engineering — such as engines, phase transitions, chemical reactions,transport phenomena, and even black holes. Many professionals teaching set it as basic theoretical courses in university. In conventional class, thermodynamics teaching usually from the viewpoint of physical chemistry, and the energy transformation system generally defined as gas balloon, cylinder, steam engine, or a reaction container, all around of them as surroundings . In response to the energy crisis, green production and sustainable development, this paper discussion on the industrial process defined as system, and natural environment as as surroundings, analysis input and output of energy transfer in industrial process, energy flow and conversion efficiency, let students learning and thinking the basic thermodynamic laws from a more macro perspective of industrial ecosystem, combining theory with Practice, enable students to better grasp and understand the basic laws of thermodynamics, make the the knowledge of class teaching meet the demand of the development of the times.
\end{abstract}

\section{Introduction}

Thermodynamics is one of the most important subject in university knowledge system of teaching, results of thermodynamic calculations are essential for other fields of physics and forchemistry, chemical engineering, aerospace engineering, mechanical engineering, cell biology, biomedical engineering, and materials science-and useful in other fields such as economics[1]. Many universities set this curriculum as basic theory courses in relative professional teaching. In our university, from 2003 this curriculum has started teaching as a basic course on ecology and environment engineering, but initially, the thermodynamics of heat engines concerned mainly the thermal properties of their 'working materials', such as steam. Chemical thermodynamics studies the role of entropy in chemical reactions. Also, statistical thermodynamics, or statistical mechanics, gave explanations of macroscopic thermodynamics by statistical predictions of the collective motion of particles based on the mechanics of their microscopic behavior[2]. At first we like other majors, the thermodynamics teaching contents mainly focus on the typical and classic principle of thermodynamics. Basic for thermodynamics are the concepts of system and surroundings. The surroundings of a thermodynamic system are other thermodynamic systems that can interact with it. An example of a thermodynamic surrounding is a heat bath, which is considered to be held at a prescribed temperature, regardless of the interactions it might have with the system. There are two fundamental kinds of entity in thermodynamics, states of a system, and processes of a system. This allows two fundamental approaches to thermodynamic reasoning, that in terms of states of a system, and that in terms of cyclic processes of a system[3]. In conventional class teaching, thermodynamics principle teaching usually from the viewpoint of physical chemistry, and the energy transformation system generally defined as gas balloon, cylinder, steam engine, or a reaction container, all around of them as surroundings. The rules of energy conversion to the state and process of system generally focused on these system as example in the class. For the study of ecology and environmental engineering students it could be found that It is difficult for them to combine the ecological and environmental system with the basic principles of thermodynamics and with their succedent learning knowledge of major courses. This paper discuss and presents the new knowledge hierarchy of thermodynamic for students learning in the class from ecological view point, using foundations of 
that theory of ecological complexity principles and summarizes the results from a review of a number of papers using thermodynamic principles to explain ecological observations especially the industry producing process which is defined as the study of industrial problems with ecological perspective.

\section{Teaching thermodynamics principle through industrial ecological knowledge}

In the class, before teaching principle especially the first law of thermodynamics, give them questions to stimulate the students interest thinking how to apply thermodynamic methods and concepts to ecology, how to describe the ecosystem's behaviour in terms of thermodynamics law, what kind of principle and criteria can be used for reveal the energy transfer and conservation impact on ecosystems? These questions will guide the students to establish the connections of classical thermodynamics theory and ecological system together, then teach the new knowledge joint analysis of industrial and ecological systems, use thermodynamics to exploit the synergy between methods from process systems engineering, systems ecology, and life cycle assessment to overcome the shortcomings of methods from each field. Together, the emergy and exergy analysis can be provided insight into the environmental performance and sustainability of the industrial process or product. This is quoting industrial ecological knowledge as teaching contour line, combining the basic principles of thermodynamics knowledge together, enable the student to use specific ecological system as the object to understand the laws of thermodynamics. The constructed teaching knowledge system of relationship between the first law of thermodynamics and the industrial and ecological system is shown in Fig.1.

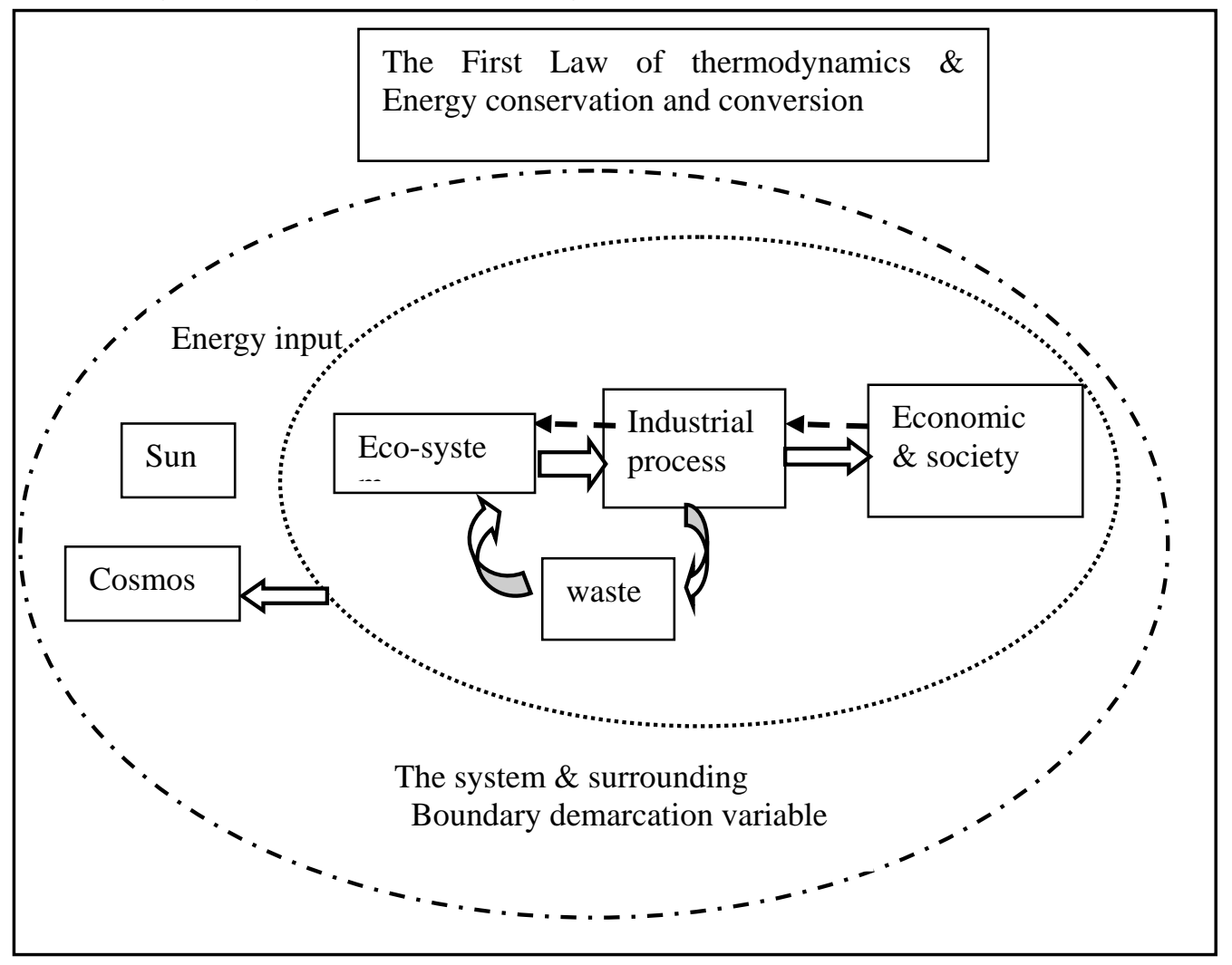

Fig.1 Teaching Thermodynamic First Law by new Knowledge

This approach considers inputs from both ecological and economic resources, as well as the impact of emissions. The approach is based on the fact that growth and sustenance of both industrial and ecological processes are limited by the available energy and its conversion to useful work. Thus, the embodied energy (emergy), that is, the energy used directly or indirectly to make a product or service is a thermodynamic measure of ecological investment or cost, while exergy loss provides a holistic measure of the impact of emissions. Through this proposed framework, it make students are 
easier to understand and grasp the basic laws of thermodynamics.

\section{Teaching energy flow rules by the metabolism of the ecosystem}

Ecological systems use flows of energy and mass to remain in a self-organized state that is far from equilibrium.The ultimate driving force for the development of ecosystems is solar energy. Even materials such as nutrients in soil, water and oxygen, industry manufactory process are all generated directly or indirectly by the capture and transformation of solar energy. Thus, ecosystems may be considered to be networks of energy flow, and all ecological products and services are transformed and stored forms of solar energy. Since ecosystems are networks of energy flow, thermodynamic methods have been popular for their analysis and modeling. Also the analysis of ecosystem energy flow is a very good teaching example of understanding the relative laws of thermodynamics, as the same time understanding the behavior and functioning of ecological. Ecosystems use the flow of energy to self-organize and remain far from equilibrium. While the typical expression of three laws of thermodynamics do not explain the behavior of ecosystems and other self-organized systems, significant research has focused on determining the thermodynamic goal functions or organizing principles that determine the development and growth of such systems, like many techniques in process systems engineering, giving examples of many techniques used in systems ecology based on the principles of thermodynamics,

Link between systems engineering and systems ecology permits the development of the proposed framework for the combined analysis of industrial and ecological systems, the energy flow in the different ecosystem like industrial system even economic society system could be defined by the variable boundary and surrounding.This is very helpful for teaching student to understand first law of thermodynamics about the energy conservation and transfer.

\section{Teaching Second Law of Thermodynamics by entropy analysis ecosystem}

Entropy is the core concept of the second law of thermodynamics, teaching experience shows that it is very difficult for students to understand the definition of entropy and usually can not grasp the true meaning of entropy function. However compared to the system used with the engine thermal process, with the ecosystem process analysis, the understanding entropy becomes more simple and more vivid. Schrodinger said that living systems maintain themselves stationary at a fairly high level of orderliness by "sucking orderliness from the environment”. Many goal functions in the ecological system include maximization of entropy production , maximization of energy dissipation, and maximization of exergy storage. These goal functions indicate that ecological and other self-organized systems create order by maximizing disorder (entropy production) in the surroundings. In teaching class using ecological system, it is easy for students learn ecosystem rules in similar with thermodynamic terms.

In the typical thermodynamic or physical chemistry texts, The second law of thermodynamics is defined as the tendency for energy to spontaneously disperse within a thermodynamic system, or from a thermodynamic system to its surrounds, unless it is hindered from doing so. Thermodynamic entropy is then defined as the measure of the energy so dispersed, at a given temperature. Entropy is an extensive property of thermodynamic systems, with units of measure of joules per degree kelvin $(\mathrm{J} / \mathrm{K})$. Extensive property means that the value of this property is proportional to the amount of matter in the system. In contrast, temperature is an intensive property: if a system is divided into two equal parts without any other changes occurring, the temperature of each part will be the same as the original system. The entropy of each part will be proportional to the amount of matter in each part, and the total entropy of the two parts will be the same as for the original system. Whenever a system comprising a specific substance or mixture of substances is in the same state (for instance, the temperature and pressure of a quantity of gas are the same everywhere at one time relative to another time), its entropy is the same, regardless of how the system came to be in that state[4,5].Within an ecosystem, there is similarly growth and decay, with individual organisms growing and decaying in cascading waves within the overall cycle of growth and decay of the 
ecosystem. And so on with systems of ecosystems through time, with, according to many researchers, an overall pattern or tendency for net complexification or growth in interrelationship at the level of nested systems of systems.[4,6] In all cases, though, this increase in complexity (defined here as increased intricacy of patterning and interrelationship between system components) is accompanied by net dispersal of energy, and increasing entropy of all constituent and encompassing thermodynamic systems.

Also society industrial-age systems driven by human agency, net increase in entropy (the entropy of my hot water system and its surrounding environment increases as water is heated), with some local decrease in entropy (the entropy of the contents of my refrigerator reduces as the temperature reduces), along with complexification of material forms- communications networks, wind farms, transport systems, information systems, buildings-and breakdown of material forms-carbon dioxide from oil, coal and gas, wear of machines, discarding of consumer goods and packaging, disposal of mine tailings and industrial waste. Growth and decay, in cascading waves within the overall cycle of growth and decay of systems[4]. By analysis the process of human industrial manufacture process as system, it could give students more practical example to understand the complex law of thermodynamics easily, as well as enhance environmental protection and energy saving consciousness.

\section{Conclusion}

The University courses teaching of theoretical basis curriculum should be combined with major knowledge. Differ from conventional class, In response to the energy crisis, green production and sustainable development, defining the ecosystem and industry process as system and natural environment as as surroundings, it is necessary letting students to learn and think the basic thermodynamic laws from a more macro perspective of ecosystem, combining theory with Practice, enable students to better grasp and understand the basic laws of thermodynamics.

\section{Acknowledgement}

The author gratefully acknowledge the financial support of the Teaching and education project (Improving the teaching methods of theory and practice for ecology thermodynamics course, 00008720, JG2012M13) of University of Science and Technology, Beijing.

\section{References}

[1] Y. Zhua, A.Q Zhang, C.X. Wang, Z.M. Nie. On strategies for e-education development under ecological thinking. Paradigm[J]. Procedia Social and Behavioral Sciences, 20102 735-2738.

[2] J.M. Polimeni. Graduate education in ecological economics[J]. Ecological Economics, 200451 287-29.

[3] S. Kirova, S. S. Veselinovska. An attempt of integration of teaching contents of the subjects ecological education and English as a foreign language[J]. Procedia Social and Behavioral Sciences, 201115 1220-1225.

[4] J. Floyd. Thermodynamics, entropy and disorder in futures studies[J]. Futures, 200739 10291044.

[5] S.E. Jørgensena, B.D. Fath. Application of thermodynamic principles in ecology[J]. Ecological Complexity, 20041 267-280.

[6] R. Ayres. Eco-thermodynamics: economics and the second law[J]. Ecological Economics, 1998 26 89-209. 